\title{
On Generalized Optimal Scheduling of High Data-Rate Bursts in CDMA Systems
}

\author{
Vincent K. N. Lau, Senior Member, IEEE, and Yu-Kwong Kwok, Senior Member, IEEE
}

\begin{abstract}
In a code-division multiple access (CDMA)-based wireless communication system, forward link is power limited and reverse link is interference limited. With power control and statistical multiplexing, voice services can be supported reasonably well. However, for high data-rate services, a more comprehensive scheduling mechanism is needed in order to achieve a high capacity while satisfying the forward and reverse link constraints. In this paper, we formulate the high data-burst scheduling as a integer programming problem using a generic CDMA system model. We also suggest an optimal algorithm for generating scheduling solutions. With cdma2000 system details plugged in the proposed algorithm, it is found that our algorithm considerably outperforms several fast heuristics, including equal sharing, first-come-first-served, longest delay first, and shortest burst first.
\end{abstract}

Index Terms-Burst scheduling, code-division multiple access (CDMA), high data rate (HDR), optimal algorithm.

\section{INTRODUCTION}

C ODE-DIVISION multiple-access (CDMA) systems have been successful in providing high-quality voice services with a high system capacity [15]. This success is largely due to the high spectrum efficiency in the statistical multiplexing of voice services which have homogeneous bandwidth requirements. In future wireless communication systems, however, high data-rate (HDR) services [1], [4], [10], [13], [14] are widely envisioned to be in great demand in supporting new mobile computing applications. These HDR services have to be efficiently provisioned [5]. In a typical CDMA system, there will not be a large number of HDR requests served simultaneously because of their high bandwidth requirements. Thus, it is not feasible to achieve the near-perfect statistical multiplexing, as in the case of voice requests. The problem, therefore, becomes the scheduling of a set of outstanding HDR requests with the goal of achieving a high capacity and/or low outage probability. By scheduling, we mean the selection of a subset of requests and the allocation of channel resources (and the burst durations of the requests) to these selected requests.

Paper approved by L. Wei, the Editor for Wireless CDMA Systems of the IEEE Communications Society. Manuscript received January 25, 2001; revised September 24, 2001; December 13, 2001; and June 25, 2002. This work was supported in part by Committee on Research Conference Grants (CRCG) research initiation grants from the University of Hong Kong under Contract 10203010 and Contract 10203413, and in part by a grant from the Hong Kong Research Grants Council under Contract HKU 7024/00E. This paper was presented in part at the 2001 IEEE Vehicular Technology Conference, Atlantic City, NJ, October 2001.

The authors are with the Department of Electrical and Electronic Engineering, The University of Hong Kong, Hong Kong (e-mail: knlau @ eee.hku.hk; ykwok@eee.hku.hk).

Digital Object Identifier 10.1109/TCOMM.2003.809258
Currently, the HDR burst scheduling problem is largely handled by simple methods such as equal sharing (ES) and firstcome-first-served (FCFS) approaches [2], [3], [6], [12]. In this paper, as detailed in Section II, we formulate the scheduling problem in an integer programming setting, with constraints induced by the forward link power limits, reverse link interference limits, and burst durations, for a generic CDMA system. With the proposed formulation, we suggest a novel optimal algorithm for obtaining scheduling solutions in Section III. With detailed cdma2000 system parameters [7], [8] plugged in our model, we simulate the scheduling mechanism and compare our proposed optimal algorithm with several fast heuristic approaches. The results, as described in Section IV, show that the optimal approach considerably outperforms the heuristic approaches. We provide some concluding remarks in Section V.

\section{Burst Scheduling PROBlem}

Suppose we have $N_{d}$ outstanding requests to be scheduled for the next frame. To serve an HDR request $j\left(j \in 1, \ldots, N_{d}\right)$ and compute the number of channels $m_{j}$ for this request, the system first has to determine its impact on the forward link power and the reverse link interference, not only for the host cell but also the neighbor cells. For the reverse link, we also need to consider the effect of soft handoff. In this section, we analyze the forward link first, followed by the reverse link.

Consider cell $k$ (which may or may not be the host cell) and let the power required to support one channel (or a single code) for request $j$ be $P_{j k}$, which can be obtained by power level measurements at the mobile terminal. If the current forward link power level is $P_{k}$ and the maximum allowable power is $P_{\max }$, then the constraint governed by the forward link for all requests is

$$
\sum_{j=1}^{N_{d}} m_{j} P_{j k} \leq P_{\max }-P_{k} .
$$

In the reverse-link case, the situation can be analyzed under two scenarios: a cell $k$ in soft handoff with the request $j$ (e.g., the host cell) and a cell $k^{\prime}$ not in soft handoff with request $j$. Let us consider the former scenario first. Similar to the forward-link case, the extra interference $I_{j k}$ introduced by the request $j$ is given by

$$
I_{j k}=m_{j} \mathcal{L}_{j k}
$$

where $\mathcal{L}_{j k}$ is the reverse link received power at base station $k$ for one channel, which depends on the processing gain, current total received power, and the received bit energy-to-interference ratio. However, it is not possible to have measurements of the 
received bit energy-to-interference ratio, and thus, we make use of the reverse link pilot strength $s_{j k}^{\mathrm{RL}}$ and the transmit power ratio of the data channel and the pilot channel $\xi_{j}$ of request $j$. We have

$$
\mathcal{L}_{j k}=\xi_{j} s_{j k}^{\mathrm{RL}} I_{k} .
$$

Thus, for a cell $k$ in soft handoff with request $j$, the extra interference $I_{j k}$ is

$$
I_{j k}=m_{j} \xi_{J} s_{j k}^{\mathrm{RL}} I_{k} .
$$

For a cell $k^{\prime}$ not in soft handoff, the major difference is that it cannot obtain the reverse link pilot strength $s_{j k^{\prime}}^{\mathrm{RL}}$. Fortunately, it is possible to estimate it using the forward link pilot strength using the relative path loss (given by the ratio of the forward link pilot strength of the cell $k^{\prime}$ and a soft handoff cell $k$ ) as follows:

$$
s_{j k^{\prime}}^{\mathrm{RL}}=s_{j k}^{\mathrm{RL}} \frac{s_{j k^{\prime}}^{\mathrm{FL}}}{s_{j k}^{\mathrm{FL}}} .
$$

Thus, the extra interference $I_{j k^{\prime}}$ is given by

$$
I_{j k^{\prime}}=m_{j} \xi_{j} s_{j k}^{\mathrm{RL}} \frac{s_{j k^{\prime}}^{\mathrm{FL}}}{s_{j k}^{\mathrm{FL}}} I_{k^{\prime}} .
$$

In summary, for the reverse link, the constraint can be expressed as

$$
\sum_{j=1}^{N_{d}} I_{j k} \leq I_{\max }-I_{k}
$$

where $I_{j k}$ is given by (4) if $k$ is a soft handoff cell, and by (6) if $k$ is not a soft handoff cell.

There is one more constraint governed by the burst duration. Because of the scheduling overhead, the duration of an HDR burst should not be too short, possibly bounded by the minimum duration $\mathcal{T}$ In other words, for a given request data size $\mathcal{B}_{j}$, the number of channels allocated to a request should be bounded by a maximum

$$
m_{j} \leq \frac{\mathcal{B}_{j}}{\mathcal{T} r}
$$

where $r$ is the rate of one channel.

Now, with the three sets of constraints given by (1), (7), and (8), we can optimize (e.g., maximize) any linear objective function $\mathcal{F}$ of the following form:

$$
\mathcal{F}(\vec{m})=\sum_{j=1}^{N_{d}} f_{j} m_{j}
$$

where $f_{j}$ can be defined such that the physical significances (e.g., fairness, delay, etc.) of the optimization is captured. As an example, we use the following definition in our study:

$$
f_{j}=\Delta_{j}+\lambda\left(w_{j}\right)^{\beta}
$$

where $\Delta_{j}$ is used for capturing the different priority of different request types (e.g., video, text data, etc.), $w_{j}$ is the waiting time of the request, and $\lambda$ and $\beta$ are positive scaling factors. Using such a function, the request that is a of a higher priority (e.g., video) and/or has waited for a long time will tend to get assigned a larger value of $m_{j}$, in order to maximize the function. Values of $\Delta_{j} \lambda$ and $\beta$ will be given in Section IV when we describe our simulation results.

\section{OPTIMAL ALGORITHM}

In this section, we derive the optimal solution to the HDR burst-scheduling problem as formulated in Section II. To simplify the derivation, we abstract out the details of the constraints and denote them as follows:

$$
\sum_{j=1}^{N_{d}} a_{j k} m_{j} \leq \mu_{k} .
$$

We tackle the problem using a divide-and-conquer approach. First, observe that the objective function (maximization) defined in (9) can be inductively expressed as

$$
\mathcal{F}(\vec{m})=\max \left\{0, \max _{j}\left[f_{j}+\mathcal{F}\left(\vec{m}-\overrightarrow{u_{j}}\right)\right]\right\}
$$

where $\overrightarrow{u_{j}}$ is a column vector in which the $j$ th element is one and all other elements are zero. Intuitively, at each step, one unit of resource (a channel) is allocated to the request contributing the most to maximizing the objective function. The key step in solving the optimization problem (12) is to partition the resource space properly, such that $\mathcal{F}(\vec{m})$ is constant within each partition. Define the resource interval $\mathcal{R}_{k}=\left[0, \mu_{k}\right]$. The resource space $\mathcal{R}_{+}^{K}$ is given by

$$
\mathcal{R}_{+}^{K}=\mathcal{R}_{1} \times \mathcal{R}_{2} \times \cdots \mathcal{R}_{K}
$$

where $K$ is the number of cells in the system.

Theorem 1: Define the set $\mathcal{A}_{j}=\left\{\left(\mu_{1}, \ldots, \mu_{K}\right): \mu_{k} \geq\right.$ $\left.a_{j k}, \forall k \in[1, K]\right\} \bigcap \mathcal{R}_{+}^{K}$. The resource space $\mathcal{R}_{+}^{K}$ can be completely partitioned by the set of nonoverlapping subspaces, $\mathcal{P}$, given by

$$
\begin{aligned}
\mathcal{P}=\left\{\mathcal { S } \left(0,[), \mathcal{S}\left(1,\left[j_{1}\right]\right), \mathcal{S}\left(2,\left[j_{1}, j_{2}\right]\right), \ldots, \mathcal{S}\left(N_{d},\left[j_{1}, \ldots, j_{N_{d}}\right]\right)\right.\right. \\
\left.\quad: j_{n} \in\left[1, N_{d}\right], j_{1} \neq j_{2} \neq \ldots \neq j_{N_{d}}\right\}
\end{aligned}
$$

where $\mathcal{S}\left(0,[)=\mathcal{R}_{+}^{K}-\bigcup_{j=1}^{N_{d}} \mathcal{A}_{j}\right.$ and $\mathcal{S}\left(n,\left[j_{1}, \ldots, j_{n}\right]\right)$ is given by

$$
\mathcal{S}\left(n,\left[j_{1}, \ldots, j_{n}\right]\right)=\bigcap_{i=1}^{n} \mathcal{A}_{j_{i}}-\bigcup_{j \notin\left[j_{1}, \ldots, j_{n}\right]} \mathcal{A}_{j} .
$$

Note that complete partition means that:

- $\bigcup_{n \in\left[0, N_{d}\right],\left[j_{1}, \ldots, j_{n}\right] \in \mathcal{C}_{n}^{N_{d}}\left(\left[1, \ldots, N_{d}\right]\right)} \mathcal{S}\left(n,\left[j_{1}, \ldots, j_{n}\right]\right)=$ $\mathcal{R}_{+}^{K}$, where $\mathcal{C}_{n}^{N_{d}}\left(\left[1, \ldots, N_{d}\right]\right)$ is the set of $n$-dimensional vector selected from the combination of the range $\left[1, \ldots, N_{d}\right]$

- $\mathcal{S}\left(n_{1},\left[j_{1}, \ldots, j_{n_{1}}\right]\right) \cap \mathcal{S}\left(n_{2},\left[j_{1}^{\prime}, \ldots, j_{n_{2}}^{\prime}\right]\right)=\Phi$ for $\mathcal{S}\left(n_{1},[) \neq \mathcal{S}\left(n_{2}, \square\right)\right.$.

Proof: Refer to Appendix A.

Note that $\mathcal{S}\left(n,\left[j_{1}, \ldots, j_{n}\right]\right)$ is called the level- $n$ partition. 
Since $\vec{\mu}$ is a real vector, we quantize the nonempty partitions of the resource space in order to facilitate the evaluation of $\mathcal{F}(\vec{m})$ in these subspaces. However, observe that

$$
\mathcal{F}(\vec{\mu} \in \mathcal{S}(0,[]))=0 \text {. }
$$

Thus, we quantize the level-1 to level- $N_{d}$ nonempty partitions of the resource space, $\mathcal{S}\left(n,\left[j_{1}, \ldots, j_{n}\right]\right) \neq \Phi$, only. Define the quantized partition, $\mathcal{Q}\left(n,\left[j_{1}, \ldots, j_{n}\right]\right) \subset \mathcal{S}\left(n,\left[j_{1}, \ldots, j_{n}\right]\right)$, as

$$
\begin{aligned}
\mathcal{Q}\left(n,\left[j_{1}, \ldots, j_{n}\right]\right) & \\
= & \left\{\left(\mu_{1}, \ldots, \mu_{K}\right) \in \mathcal{S}\left(n,\left[j_{1}, \ldots, j_{n}\right]\right)\right. \\
& \mu_{k}=0, \text { if } a_{j k}=0, \forall j \in\left[j_{1}, \ldots, j_{n}\right] \\
& \mu_{k}=\max _{j \in\left[j_{1}, \ldots, j_{n}\right]}\left[a_{j k}\right]+i \delta_{q_{n}}, \text { for some } i \in[0,1, \ldots] ; \\
& \text { if } \left.a_{j k} \neq 0 .\right\}
\end{aligned}
$$

where $\delta_{q_{n}}$ is the quantization interval for level- $n$ partition.

We solve (12) for $\vec{\mu}$ in each element of the quantized partition set $\mathcal{P}$. The sequence of evaluation of $\mathcal{F}(\vec{m})$ starts from the zerolevel partition $\vec{\mu} \in \mathcal{S}(0,[)$ and then the first-level quantized partition $\vec{\mu} \in \mathcal{Q}\left(1,\left[j_{1}\right]\right)$, the second-level quantized partition $\vec{\mu} \in \mathcal{Q}\left(2,\left[j_{1}, j_{2}\right]\right)$, and so on, because $\mathcal{F}$ can be expressed in terms of already found $\mathcal{F}$ at lower level partitions. For any $\vec{\mu} \in$ $\mathcal{Q}\left(n,\left[j_{1}, \ldots, j_{n}\right]\right)$, we have

$$
\left.\mathcal{F}(\vec{m})=\max _{j \in\left[j_{1}, \ldots, j_{n}\right]}\left\{f_{j}+\mathcal{F}\left(\mathcal{G}_{n}\left[\vec{m}-\vec{u}_{j}\right]\right)\right]\right\}
$$

Note that $\hat{\vec{x}}=\mathcal{G}_{n}[\vec{x}]$ is the quantized vector, such that

$$
\begin{aligned}
|\vec{x}-\hat{\vec{x}}| \leq & |\vec{x}-\vec{y}|, \forall \vec{y} \in \mathcal{S}(0,[]) \\
& \times \bigcup_{r=1}^{n} \bigcup_{\left(p_{1}, \ldots, p_{r}\right) \in \mathcal{C}_{r}^{n}\left(\left[j_{1}, \ldots, j_{n}\right]\right)} \mathcal{Q}\left(r,\left[p_{1}, \ldots, p_{r}\right]\right)
\end{aligned}
$$

where $\mathcal{C}_{r}^{n}\left(\left[j_{1}, \ldots, j_{n}\right]\right)$ is the combinatorial set given by

$$
\mathcal{C}_{r}^{n}\left(\left[j_{1}, \ldots, j_{n}\right]\right)=\left\{\left(p_{1}, \ldots, p_{r}\right): p_{i} \in\left[j_{1}, \ldots, j_{n}\right], p_{i_{1}} \neq p_{i_{2}}\right\}
$$

Denote the allocation index, $j^{*}$ as $j^{*}=g(\vec{\mu})$ where $j^{*}$ is the result of the max operation in (18). The allocation vector, $h(\vec{\mu})$, is defined as $\left(m_{1}, m_{2}, \ldots, m_{N_{d}}\right)$ such that $m_{j^{*}}=1$ and $m_{j^{\prime}}=$ $0, \forall j^{\prime} \neq j^{*}$.

Define the initial resource vector, $\vec{\mu}_{1}$ as $\mathcal{G}_{N_{d}}\left[\left(P_{\max }-\right.\right.$ $\left.\left.P_{1}\right),\left(P_{\max }-P_{2}\right), \ldots,\left(P_{\max }-P_{K}\right)\right] . \mathcal{F}\left(\vec{\mu}_{1}\right)$ has been found with the first allocation index $j_{1}^{*}=g\left(\vec{\mu}_{1}\right)$. The next resource vector, $\vec{\mu}_{2}=\vec{\mu}_{1}-\vec{u}_{j_{1}^{*}}$. Thus, the second allocation index, $j_{2}^{*}=g\left(\vec{\mu}_{2}\right)$. In general, we have the $p$ th resource vector and the $p$ th allocation index given by

$$
\begin{aligned}
\vec{\mu}_{p} & =\vec{\mu}_{p-1}-\vec{u}_{j_{p-1}^{*}} \\
j_{p}^{*} & =g\left(\vec{\mu}_{p}\right)
\end{aligned}
$$

TABLE I

SIMULATION PARAMETERS

\begin{tabular}{ll}
\hline Parameter & Value \\
\hline$\Delta_{j}$ (voice) & 5.0 \\
$\Delta_{j}$ (video) & 10.0 \\
$\Delta_{j}$ (data) & 1.0 \\
$\lambda$ & 4.0 \\
$\beta$ & 2.0 \\
path loss exponent & 4 \\
average adjacent cell load & $50 \%$ \\
chip rate & $7.3728 \mathrm{Mcps}$ \\
FCH rate & $14.4 \mathrm{kbps}$ \\
cdma2000 SCH rate & {$[14.4 \mathrm{kbps}-1.8432 \mathrm{Mbps}]$} \\
processing gain (cdma2000-FCH) & 512 \\
processing gain (cdma2000-SCH) & {$[512-4]$} \\
BER (FCH) & $10^{-2}$ \\
BER (SCH) & $10^{-4}$ \\
$E_{s} / I_{0}(\mathrm{cdma} 2000-\mathrm{FCH})$ & $7 \mathrm{~dB}$ \\
$E_{s} / I_{0}(\mathrm{cdma} 2000-\mathrm{SCH})$ & $13 \mathrm{~dB}$ \\
\hline
\end{tabular}

where the iteration stops when $\vec{\mu}_{p_{0}} \in \mathcal{S}\left(0,[)\right.$ for some $p_{0}$. The resulting optimal allocation is given by $\sum_{p=1}^{p_{0}} h\left(\vec{\mu}_{p}\right)$.

A limitation of our optimal approach is that in the worst case, the total number of partitions in the partition set $\mathcal{P}$ is $2^{N_{d}}$. Thus, the computational complexity of the above algorithm is exponential in $N_{d}$ in the worst case.

\section{Simulation Results}

Simulating the cdma2000 environment, the proposed optimal burst scheduling algorithm is compared with several fast heuristics: ES, FCFS, longest delay first (LDF), and shortest burst first (SBF). All the burst-scheduling algorithms are evaluated based on static and dynamic simulations used in [9]. Specifically, we employ static Monte Carlo techniques for generating the coverage results for high-speed data requests. The cellular architecture is assumed to be a hexagonal grid with base stations (sectorized with three sectors) located at the center of every hexagon. Distance loss is modeled by $d^{-\delta}$, where $\delta$ is the propagation constant. Shadow fading is modeled using the Mawira model [11] for an urban environment. We repeat the Monte Carlo simulations 100 times to obtain the static coverage results. Throughout all experiments, we assume adjacent cell load is $50 \%$. Note that for parameters $\Delta_{j}, \lambda$, and $\beta$, one should set them to suit the goals to be achieved. For example, if the primary goal is to minimize the delay, then the parameters $\lambda$ and $\beta$ should have large values. On the other hand, if traffic type is a more important concern (e.g., some data requests are privileged), the value of $\Delta_{j}$ should be increased appropriately to reflect the relative importance. In our experiments, we try to strike the balance between the two objectives. Finally, in order to apply our modeling in a cdma2000 system, we need to augment the model with some specific parameters. The HDR channels, namely the SCHs (supplemental channels), are in fact different from the FCH (fundamental channel) in the cdma2000 system. Thus, we need to use an adjustment factor $\alpha_{j}^{\mathrm{FL}}$ to compute the extra power required in (1). Similarly, another adjustment factor needs to be used for the calculation of extra interference in (7). Table I summarizes the parameters used. 


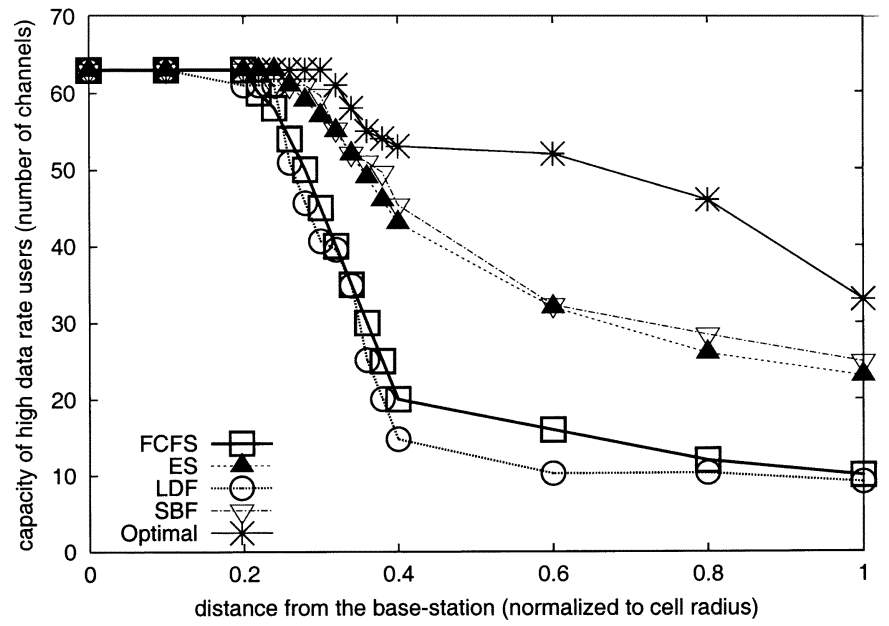

Fig. 1. Number of channels available on the forward link with varying distances from the base station.

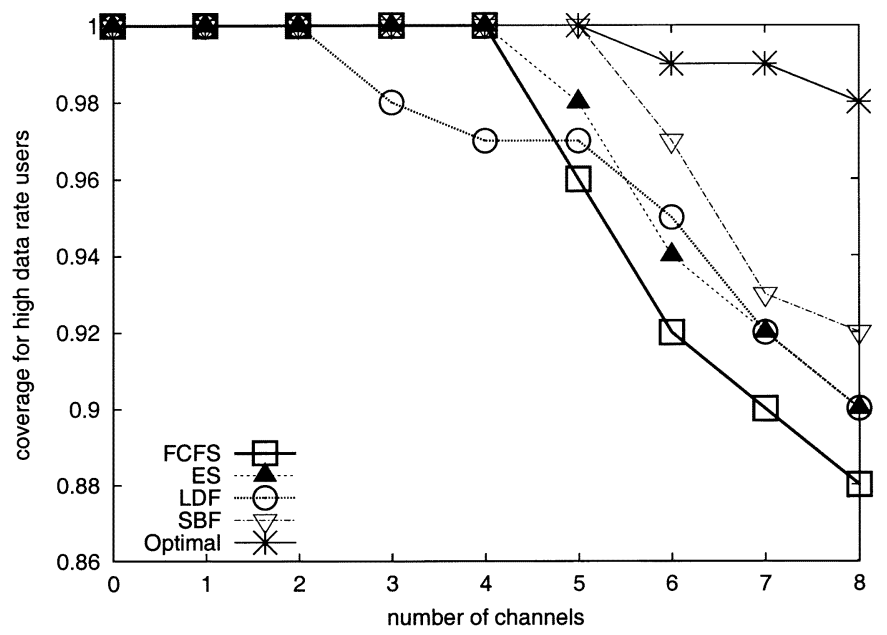

Fig. 2. Forward-link coverage area as a function of number of channels.

We use a simple model for the mobility of the terminals. Each terminal (voice or data) selects a random starting position, which is uniformly distributed over the cell. The direction of motion is also randomly selected. The motion is rectilinear until a call is finished. The speed of motion is assumed to be a constant $(35 \mathrm{mi} / \mathrm{h})$. Soft handoff and perfect power control are assumed also. To obtain results of dynamic measurements such as admission and outage probabilities, we performed ten independent simulations with 5000 calls each.

Fig. 1 shows the forward-link capacity (in terms of the number of FCH channels) of the five different burst-scheduling schemes. Here, the load by the voice requests in the cell is also $50 \%$. As can be seen, the proposed optimal algorithm outperforms the four heuristic approaches by a considerable margin. This clearly demonstrates that if the extra processing requirements are affordable, optimizing the HDR bursts scheduling is highly beneficial. Furthermore, it can be seen that two of the heuristic approaches, LDF and SBF, exhibit rather fluctuating behaviors. This is due to the fact that their scheduling decisions very much depend on the source data arrivals. For example, if there is a sudden accumulation of data making the delays longer for some terminals, the scheduler may select terminals with a

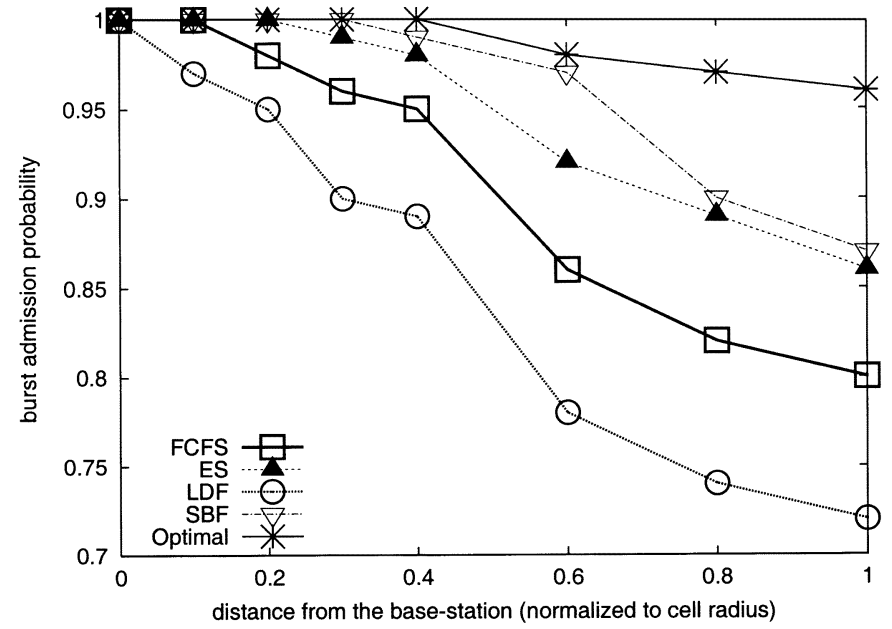

Fig. 3. Average admission probabilities on the reverse link.

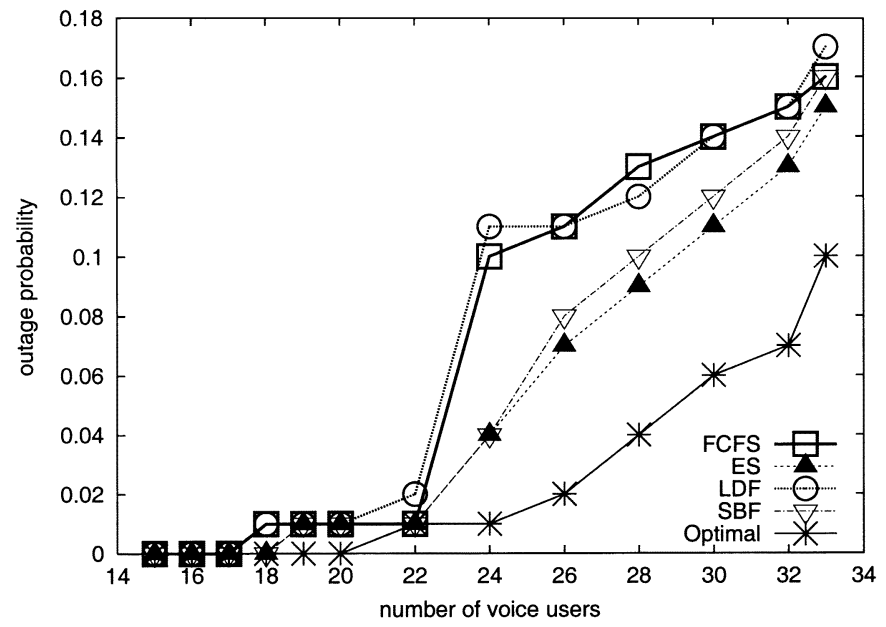

Fig. 4. Outage probabilities for voice requests.

higher resource demand. Similarly, for SBF, a terminal with a smaller amount of residual data may get served earlier than it should be. On the other hand, as expected, the high capacity is not available throughout the entire cell but just the area near the base station, as indicated in Fig. 2 (in these results, there is one HDR request and the voice requests load in the cell is 50\%).

For the reverse link, we obtain results on admission and outage probabilities, as shown in Figs. 3 and 4. For the results on admission probabilities, the requested data rate is $57.6 \mathrm{~Kb} / \mathrm{s}$ and the voice load in the cell is $50 \%$. The results on outage probabilities are obtained by dynamic simulations and there is one data request (with rate $57.6 \mathrm{~Kb} / \mathrm{s}$ ). Again, we can see that the optimal approach can allow a high admission probability, through its judicious merging of different requests arrived at different time periods. The optimal approach can also support a much higher number of voice requests.

\section{CONCLUSION}

In this paper, we propose an integer programming formulation of the HDR burst-scheduling problem in a CDMA system. Our generic model considers the forward link power limits and reverse link interference limits. We also suggest a novel optimal 
algorithm for obtaining high-quality scheduling solutions. Simulating a cdma2000 system, we have found that the optimal algorithm significantly outperforms several fast heuristic approaches. However, as obtaining optimal solutions requires a much higher time complexity, further research should be done in devising a fast optimization algorithm which can generate near-optimal solutions.

\section{APPENDIX}

\section{A. Proof of Theorem 1}

We have to prove the following results for complete partitioning:

$$
\begin{array}{r}
\bigcup_{n \in\left[0, N_{d}\right],\left[j_{1}, \ldots, j_{n}\right] \in \mathcal{C}_{n\left(\left[1, \ldots, N_{d}\right]\right)}^{N_{d}}} \mathcal{S}\left(n,\left[j_{1}, \ldots, j_{n}\right]\right)=\mathcal{R}_{+}^{K} \\
\mathcal{S}\left(n_{1},\left[j_{1}, \ldots, j_{n_{1}}\right]\right) \bigcap \mathcal{S}\left(n_{2},\left[j_{1}^{\prime}, \ldots, j_{n_{2}}^{\prime}\right]\right)=\Phi
\end{array}
$$

To prove (23), we consider the following backward induction formulation. At the $N_{d}$ th step, we have $\mathcal{S}\left(N_{d},\left[1, \ldots, N_{d}\right]\right)$ given by

$$
\mathcal{S}\left(N_{d},\left[1, \ldots, N_{d}\right]\right)=\underset{\left[j_{1}, \ldots, j_{N_{d}}\right] \in \mathcal{C}_{N_{d}}^{N_{d}}}{\bigcup}\left[\bigcap_{p=1}^{N_{d}} \mathcal{A}_{j_{p}}\right]=\left[\bigcap_{j=1}^{N_{d}} \mathcal{A}_{j}\right] .
$$

At the $r$ th step, we have

$$
\begin{aligned}
& {\left[\bigcup_{\left[j_{1}, \ldots, j_{r}\right] \in \mathcal{C}_{r}^{N_{d}}\left(\left[1, \ldots, N_{d}\right]\right)} \mathcal{S}\left(r,\left[j_{1}, \ldots, j_{r}\right]\right)\right] } \\
& \times \bigcup_{\left[j_{1}, \ldots, j_{r+1}\right] \in \mathcal{C}_{r+1}^{N_{d}}\left(\left[1, \ldots, N_{d}\right]\right)}\left[\bigcap_{p=1}^{r+1} \mathcal{A}_{j_{p}}\right] \\
&=\bigcup_{\left[j_{1}, \ldots, j_{r}\right] \in \mathcal{C}_{r}^{N_{d}}}\left[\bigcap_{p=1}^{r} \mathcal{A}_{j_{p}}-\bigcup_{j=1: j \neq\left[j_{1}, \ldots, j_{r}\right]}^{N_{d}} \mathcal{A}_{j}\right] \\
&\left.\times \bigcup_{\left[j_{1}, \ldots, j_{r+1}\right] \in \mathcal{C}_{r+1}^{N_{d}}\left(\left[1, \ldots, N_{d}\right]\right)}^{r+1} \mathcal{A}_{p=1}^{r+1}\right] \\
&= \\
& \qquad \bigcup_{\left[j_{1}, \ldots, j_{r}\right] \in \mathcal{C}_{r}^{N_{d}}}\left[\bigcap_{p=1}^{r} \mathcal{A}_{j_{p}}\right] .
\end{aligned}
$$

Thus, inductively, we have

$$
\begin{aligned}
& \mathcal{S}(0,[]) \bigcup\left[\bigcup_{r=1}^{N_{d}} \bigcup_{\left[j_{1}, \ldots, j_{r}\right] \in \mathcal{C}_{r}^{N_{d}}} \mathcal{S}\left(r,\left[j_{1}, \ldots, j_{r}\right]\right)\right] \\
& =\mathcal{S}\left(0,[) \bigcup \bigcup_{r=1}^{N_{d}-1}\left[\bigcup_{\left[j_{1}, \ldots, j_{r}\right] \in \mathcal{C}_{r}^{N_{d}}} \mathcal{S}\left(r,\left[j_{1}, \ldots, j_{r}\right]\right) \bigcup\left[\bigcap_{j=1}^{N_{d}} \mathcal{A}_{j}\right]\right.\right.
\end{aligned}
$$

$$
\begin{aligned}
= & \mathcal{S}(0,[]) \bigcup \bigcup_{r=1}^{N_{d}-2}\left[\bigcup_{\left[j_{1}, \ldots, j_{r}\right] \in \mathcal{C}_{r}^{N_{d}}} \mathcal{S}\left(r,\left[j_{1}, \ldots, j_{r}\right]\right)\right] \bigcup \\
& \times\left\{\bigcup_{\left[j_{1}, \ldots, j_{N_{d}-1}\right] \in \mathcal{C}_{N_{d}-1}^{N_{d}}} \mathcal{S}\left(N_{d}-1,\left[j_{1}, \ldots, j_{N_{d}-1}\right]\right)\right. \\
& \left.\times \bigcup\left[\bigcap_{j=1}^{N_{d}} \mathcal{A}_{j}\right]\right\} \\
= & \cdots \\
= & \mathcal{S}(0,[]) \bigcup_{j=1}^{N_{d}} \mathcal{A}_{j} \\
= & \mathcal{R}_{+}^{K} .
\end{aligned}
$$

Note that the first equality in (27) follows from (25). The second-to-last equality follows by repeatedly applying (26).

We prove (26) by contradiction. Assume there exists an element $\vec{x} \in \mathcal{S}\left(n_{1},\left[j_{1}, \ldots, j_{n_{1}}\right]\right) \cap \mathcal{S}\left(n_{2},\left[j_{1}^{\prime}, \ldots, j_{n_{2}^{\prime}}\right]\right)$. Since $\mathcal{S}\left(n_{1},\left[j_{1}, \ldots, j_{n_{1}}\right]\right)=\left[\bigcap_{p=1}^{n_{1}} \mathcal{A}_{j_{p}}-\bigcup_{j=1: j \neq\left[j_{1}, \ldots, j_{n_{1}}\right]}^{N_{d}} \mathcal{A}_{j}\right]$, we have

$$
\begin{array}{ll}
\vec{x} \in \mathcal{A}_{j_{p}} & \left.\forall p \in\left[1, n_{1}\right]\right] \\
\vec{x} \notin \mathcal{A}_{j} & \forall j \neq\left\{j_{1}, \ldots, j_{n_{1}}\right\} \\
\vec{x} \in \mathcal{A}_{j_{p}^{\prime}} & \left.\forall p \in\left[1, n_{2}\right]\right] \\
\vec{x} \notin \mathcal{A}_{j} & \forall j \neq\left\{j_{1^{\prime}}, \ldots, j_{n_{2}}^{\prime}\right\} .
\end{array}
$$

Without loss of generality, assume that $j_{1} \neq\left\{j_{1}^{\prime}, \ldots, j_{n_{2}}^{\prime}\right\}$. We have from (28) and (31) that $\vec{x} \in \mathcal{A}_{j_{1}}$ and $\vec{x} \notin \mathcal{A}_{j_{1}}$. This results in contradiction.

\section{ACKNOWLEDGMENT}

The authors would like to thank the reviewers (in particular, Reviewer C) for their insightful comments which have greatly improved the presentation of this paper.

\section{REFERENCES}

[1] W. C. Chan, E. Geraniotis, and K. Etemad, "Performance analysis of ISMA for short burst data service in wireless CDMA networks," in Proc. ICC'99, 1999, pp. 1115-1120.

[2] R. P. Ejzak, D. N. Knisely, S. Kumar, S. Laha, and S. Nanda, "BALI: A solution for high-speed CDMA data," Bell Labs Tech. J., pp. 134-151, 1997.

[3] V. K. Garg, IS-95 CDMA and cdma2000. Englewood Cliffs, NJ: Prentice-Hall, 2000.

[4] N. Guo, S. D. Morgera, and P. Mermelstein, "Common packet data channel (CPDC) for integrated wireless DS-CDMA networks," IEEE J. Select. Areas Commun., vol. 14, pp. 735-749, May 1996.

[5] W. Huang and V. K. Bhargava, "Performance evaluation of a DS-CDMA cellular system with voice and data services," in Proc. PIMRC'96, 1996, pp. $588-592$.

[6] C.-L. I and S. Nanda, "Load and interference based demand assignment (LIDA) for integrated services in CDMA wireless systems," in Proc. GLOBECOM'96, 1996, pp. 235-241.

[7] D. N. Knisely, S. Kumar, S. Laha, and S. Nanda, "Evolution of wireless data services: IS-95 to cdma2000," IEEE Commun. Mag., vol. 36, pp. 140-149, Oct. 1998.

[8] D. N. Knisely, Q. Li, and N. S. Ramesh, "Cdma2000: A third-generation radio transmission technology," Bell Labs Tech. J., pp. 63-78, July-Sept. 1998. 
[9] S. Kumar and S. Nanda, "High data-rate packet communications for cellular networks using CDMA: Algorithms and performance," IEEE J. Select. Areas Commun., vol. 17, pp. 472-492, Mar. 1999.

[10] S. Manning, A. Gutierrez, and M. Wang, "A short data burst mechanism for third-generation CDMA wireless packet data," in Proc. VTC'99, 1999 , pp. $521-525$

[11] A. Mawira, "Models for the spatial correlation functions of the (log)normal component of the variability of VHF/UHF field strength in urban environment," in Proc. 3rd IEEE Int. Conf. Personal, Indoor, Mobile Radio Communications, Oct. 1992, pp. 436-440.

[12] S. Nanda, K. Balachandran, and S. Kumar, "Adaptation techniques in wireless packet data services," IEEE Commun. Mag., vol. 38, pp. 54-64, Jan. 2000

[13] R. Prasad and T. Ojanpera, "A survey on CDMA: Evolution toward wideband CDMA," in Proc. IEEE Int. Conf. Spread Spectrum Techniques and Applications, 1998, pp. 323-331.

[14] A. Sampath and J. M. Holtzman, "Access control of data in integrated voice/data CDMA systems: Benefits and tradeoffs," IEEE J. Select. Areas Commun., vol. 15, pp. 1511-1526, Oct. 1997.

[15] A. J. Viterbi, CDMA: Principles of Spread Spectrum Communication. Reading, MA: Addison-Wesley, 1995.

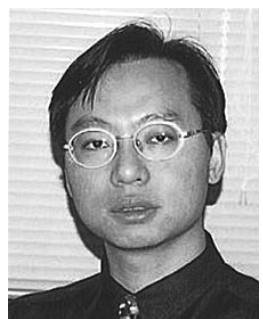

Vincent K. N. Lau (M'97-SM'01) was born in Hong Kong. He received the undergraduate degree in electrical and electronics engineering with distinction (first-class honors) from the University of Hong Kong, Hong Kong, in 1992, and the Ph.D. degree from Cambridge University, Cambridge, U.K. in 1997.

He joined Lucent Technologies, Whippany, NJ, as a Member of Technical Staff in 1997. He left Lucent and joined the University of Hong Kong in 1999 as an Assistant Professor, and was later appointed the Codirector of Information Engineering Programmes as well as the Codirector of the 3G Wireless Technology Center. He left the University and rejoined Lucent in July 2001. His research interests include digital transceiver design, adaptive modulation and channel coding, CDMA power control, soft handoff and CREST factor control algorithms, jointly adaptive multiple access protocols, and shortrange wireless ad hoc networking. He has published more than 60 papers in IEEE conference proceedings and journals, and is the author of a book chapter on wideband CDMA technologies. In addition, he has five U.S. patents pending.

Dr. Lau was the invited Session Chair of the IEEE WCNC 2000 International Conference, the IEEE CAS 3G Workshop 2000, and the SCT 2001 International Conference. He received two Best Paper Awards from the Institution of Electrical Engineers and the Hong Kong Institute of Engineers (HKIE) in 1999. He was awarded the Sir Edward Youde Memorial Fellowship and an award from the Croucher Foundation in 1995.

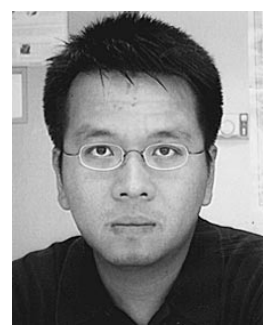

Yu-Kwong Kwok (S'94-M'95-SM'03) received the B.Sc. degree in computer engineering from the University of Hong Kong, Hong Kong, in 1991, and the M.Phil. and Ph.D. degrees in computer science from the Hong Kong University of Science and Technology (HKUST), Hong Kong, in 1994 and 1997, respectively.

$\mathrm{He}$ is currently an Associate Professor in the Department of Electrical and Electronic Engineering, University of Hong Kong. Before joining the University of Hong Kong, he was a Visiting Scholar in the Parallel Processing Laboratory, School of Electrical and Computer Engineering, Purdue University, West Lafayette, IN. His research interests include mobile computing, wireless communications, network protocols, and distributed computing algorithms.

Dr. Kwok is a member of the Association for Computing Machinery (ACM), the IEEE Computer Society, and the IEEE Communications Society. 